\title{
Flexibilität, Kosten und Industrie 4.0 im Blick
}

\author{
Die Anpassung an veränderte Prozesse und Anforderungen, kleinere Losgrößen und die \\ fortschreitende Globalisierung sind einige der Themen, die die Reinigungsbranche beschäftigt. \\ Die Bedeutung von Industrie 4.0 nimmt in der Reinigungstechnik ebenfalls zu.
}

Ob partikulär oder filmisch - das Thema Restschmutz spielt in Gesprächen im Bereich der industriellen Reinigungstechnik immer eine Rolle. Dabei sind zwei Tendenzen zu beobachten. Zum einen ist es eine kontinuierlich wachsende Zahl von Unternehmen, die Sauberkeitsgrenzwerte für Bauteile definieren - und das international. Darüber hinaus wird die Einhaltung der Grenzwerte immer häufiger und in regelmäßigeren Abständen kontrolliert. Daraus resultieren auch verstärkte Anstrengungen von Anlagenbauern, Herstellern von Messtechnik und Verbänden, Lösungen für eine inline beziehungsweise prozessnahe Kontrolle auf partikulären und filmischen Restschmutz zur Verfügung zu stellen.

Zum anderen werden Forderungen - insbesondere wenn es um partikuläre Sauberkeitsanforderungen geht - genauer hinterfragt. Denn nicht alles, was gefordert wird, ist reinigungstechnisch machbar und nicht alles, was machbar ist, erweist sich als erforderlich oder sinnvoll. Denn mit zunehmenden Sauberkeitsanforderungen steigen die Investitions- und Betriebskosten der Reinigungsanlagen.

\section{Herausforderung Globalisierung}

Um im zunehmend globalisierten Markt wettbewerbsfähig zu bleiben, sind die Anlagenhersteller auch verstärkt gefordert, hochwertige Technologien für nicht so anspruchsvolle Reinigungsaufgaben zu einem optimierten Preis-/Leistungsverhältnis anzubieten. Denn speziell in diesem Segment nimmt der Wettbewerb durch ausländische Anbieter aus Süd- und Osteuropa sowie China zu.

Die fortschreitende Globalisierung stellt die überwiegend mittelständisch geprägten Unternehmen vor weitere Herausforderungen. Dazu zählt einerseits eine möglichst breite globale Aufstellung, um die Auswirkungen lokaler und regionaler Marktschwankungen sanfter abfedern zu können. Andererseits sind bei multinational tätigen Kunden die Reinigungsprozesse an unterschiedliche länderspezifische Vorgaben und Gesetzgebungen anzupassen und dabei eine gleichbleibende Reinigungsqualität zu gewährleisten.

\section{Höhere Modularität und Anpassungsfähigkeit}

Nichts ist so beständig wie der Wandel. Um auf die Auswirkungen dieser alten Weisheit schnell und ohne großen finanziellen Aufwand reagieren zu können, wird die Zukunftsfähigkeit von Reinigungsanlagen zum immer wichtigeren Entscheidungskriterium. Die Hersteller reagieren darauf, in dem sie bei der Konzeption von Anlagen Vorkehrungen für eine spätere Anpassung beispielsweise an veränderte Teilespektren, Sauberkeitsanforderungen oder Prozesse treffen. Dies erfolgt unter anderem durch leistungsfähigere Ultraschalleinrichtungen, Druckpumpen und Filtrationssysteme. Darüber hinaus gibt es Entwicklungen, die es ermöglichen, individueller auf die Größe und Geometrie der zu reinigenden Teile zu reagieren. Auch der Austausch von Reinigungswerkzeugen wie beispielsweise Spritzmodulen ist ein Lösungsweg, ebenso wie die Trennung von Reinigung und Trocknung bei wässrigen Reinigungsprozessen. Darüber hinaus arbeiten die Anlagenbauer an der Optimierung bestehender und der Entwicklung neuer Reinigungsverfahren.

\section{Industrie 4.0 - nicht mehr nur Schlagwort}

Automatisierung und die Einbindung der Reinigung in vernetzte Prozesse spielt ebenfalls eine zunehmend wichtige Rolle bei den Anwendern von Reinigungssystemen. Beispiele sind hier adaptive Prozesse wie die kontinuierliche InlineKontrolle der Reinigungsbäder mit vollautomatischer Nachdosierung des Reinigers, eine permanente Erfassung aller Prozessparameter und deren Dokumentation oder auch die Inline-Kontrolle der erzielten Sauberkeit. Erforderlich dafür sind entsprechende steuerungstechnische und sensorische Lösungen, die teilweise bereits zur Verfügung stehen. Ein Thema sind Apps beispielsweise für Diagnose, Wartung, Ersatzteilversorgung und für Bedienungsanleitungen.

Ein nächster Entwicklungsschritt ist die Anpassung der Verfahrenstechnik an den Reinigungsbedarf der Teile. Eine Sensorik erkennt, wie stark die gerade in der Anlage befindlichen Werkstücke verschmutzt sind und passt den Prozess automatisch an. Bis eine solche intelligente Reinigungsanlage Realität ist, vergeht aber sicher noch etwas Zeit. //

Doris Schulz, Fachjournalistin, Stuttgart 